\title{
Endovascular Management of Internal Carotid Artery Pseudoaneurysm Secondary to Pediatric Deep Neck Space Infection: A Case Report and Review of Literature
}

\author{
Rengarajan Rajagopal $^{1}$ Smily Sharma ${ }^{1}$ Seenu Bagarhatta ${ }^{2}$ Sarbesh Tiwari ${ }^{1}$ Rajeev Bagarhatta ${ }^{3}$ \\ ${ }^{1}$ Department of Diagnostic \& Interventional Radiology, All India \\ Institute of Medical Sciences, Jodhpur, Rajasthan, India \\ 2 Department of Radiodiagnosis, SMS Medical College \& Attached \\ Hospitals, Jaipur, Rajasthan, India \\ Address for correspondence Rengarajan Rajagopal, MD, DM, \\ Department of Diagnostic \& Interventional Radiology, All India \\ Institute of Medical Sciences, Jodhpur 342005, Rajasthan, India \\ (e-mail: heraghava@gmail.com).
}

${ }^{3}$ Department of Cardiology, SMS Medical College \& Attached

Hospitals, Jaipur, Rajasthan, India

Arab J Intervent Radiol 2021;5:110-113.

\begin{abstract}
Keywords

- complicated tonsillitis

- internal carotid artery pseudoaneurysm

- coil embolization

Pseudoaneurysms of extracranial internal carotid artery (ICA) are rare in children. Main causes include trauma, iatrogenic causes, and neck space infection. Prompt diagnosis and management is vital, in view of life-threatening complications like fatal airway hemorrhage and stroke. Endovascular management has currently become the preferred treatment strategy due to its minimally invasive nature and lower complication rates. We report a rare case of mycotic pseudoaneurysm of extracranial ICA in a 4-yearold child as a complication of neck space infection, which was successfully managed with endovascular parent artery occlusion.
\end{abstract}

\section{Introduction}

Pseudoaneurysms of extracranial internal carotid artery (ICA) are rare lesions in children with fewer than 100 cases reported. ${ }^{1,2}$ Main causes include trauma, iatrogenic causes, infections, and malignancies with infection and trauma being the most common causes in children. ${ }^{3,4}$ Prompt diagnosis and management is vital, in view of life-threatening complications like fatal airway hemorrhage and stroke. ${ }^{2,5}$ Endovascular management has become the preferred treatment strategy due to its minimally invasive nature and lower complication rates. $^{6,7}$ We report a rare case of mycotic pseudoaneurysm of extracranial ICA in a 4-year-old child as a complication of neck space infection, which was suc- cessfully managed with endovascular parent artery occlusion (PAO).

\section{Case}

A 4-year male child presented to the emergency room with high-grade fever and tender right neck swelling for 2 days. The child had no neurological symptoms, prior history consistent with tonsillitis or surgery. Complete blood evaluation showed leukocytosis $\left(35,000\right.$ cells $\left./ \mathrm{mm}^{2}\right)$ with neutrophilia. Neck ultrasound showed significant bilateral cervical lymphadenopathy with hypoechoic lesion in the right submandibular region with internal swirling flow. Computed published online January 6, 2022
DOI https://doi.org/ 10.1055/s-0041-1740341. ISSN 2542-7075. (c) 2022. The Pan Arab Interventional Radiology Society. All rights reserved.

This is an open access article published by Thieme under the terms of the Creative Commons Attribution-NonDerivative-NonCommercial-License, permitting copying and reproduction so long as the original work is given appropriate credit. Contents may not be used for commercial purposes, or adapted, remixed, transformed or built upon. (https://creativecommons.org/ licenses/by-nc-nd/4.0/)

Thieme Medical and Scientific Publishers Pvt. Ltd., A-12, 2nd Floor, Sector 2, Noida-201301 UP, India 


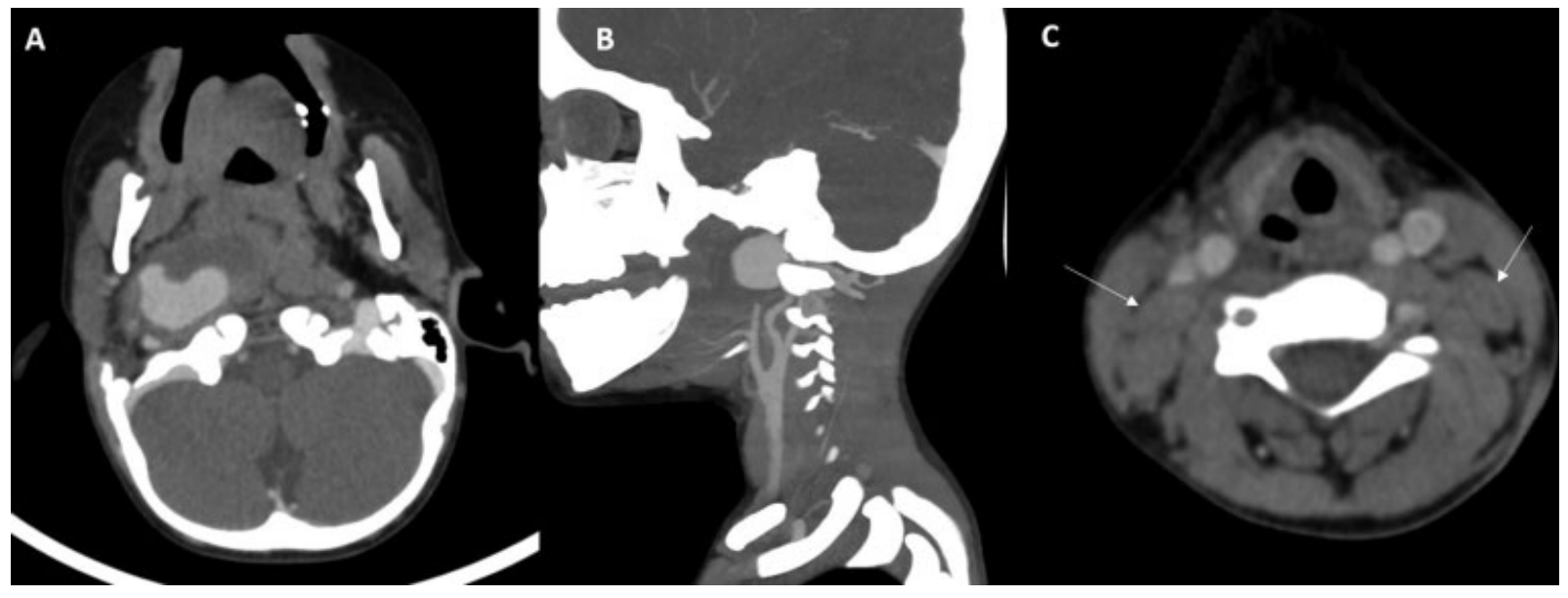

Fig. 1 Contrast-enhanced computed tomography (CECT) neck in a 4-year-old child showing saccular pseudoaneurysm from right extracranial cervical internal carotid artery (ICA) with peripheral thrombosis and enhancing walls (axial - A; sagittal - B) with prominent bilateral cervical lymphadenopathy (white arrows in C) suggestive of infection.

tomography (CT) angiography showed a saccular pseudoaneurysm from the right extracranial cervical carotid artery with peripheral thrombosis and enhancing walls (suggestive of mycotic pseudoaneurysm) close to the skull base (- Fig. 1). Adjacent airway was not compressed. Blood samples were sent for cultures. The child was admitted for intravenous antibiotics and rehydration. Within few hours of admission, the general status of the child deteriorated with purulent blood-streaked nasal discharge. With the clinical suspicion of rupture, the child was taken up for emergency endovascular PAO. Diagnostic angiograms confirmed the presence of sac-

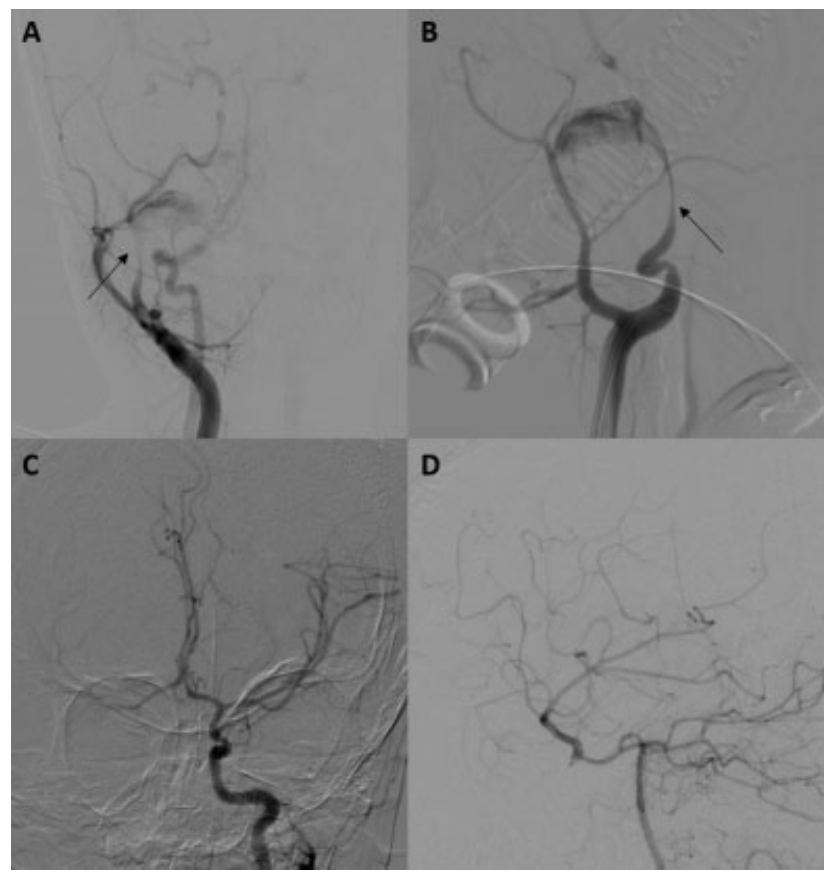

Fig. 2 Diagnostic angiogram showing contrast filling the pseudoaneurysm from cervical internal carotid artery (ICA) (black arrows in A and B) with absence of antegrade filling of distal ICA. Diagnostic angiograms showing filling of cavernous right ICA through anterior communicating artery (in C) and posterior communicating artery (in D). cular pseudoaneurysm from the right cervical ICA. On cerebral angiography, the right anterior cerebral artery and middle cerebral artery were shown to fill from left ICA and right vertebral artery with good-sized anterior communicating artery and right posterior communicating artery (-Fig. 2). Right ICA was occluded proximal to the pseudoaneurysm with coils using a vertebral diagnostic catheter (Interlock-35, $6 \mathrm{~mm} \times 100 \mathrm{~mm}$, Boston Scientific). Antibiotics (cultures isolated Staphylococcus sp.) were continued for up to 4 weeks in postprocedure period. No neurological deficits were seen at periprocedural period. Follow-up CT angiograms at 4 weeks showed thrombosed pseudoaneurysm with well-collateralized intracranial circulation ( - Fig. 3 ). The child is well at 6 months of clinical follow-up.

\section{Discussion}

ICA pseudoaneurysms are an uncommon complication of deep neck space infections especially after advancements in antibiotic therapy. These are more common in children as compared with adults due to higher incidence of deep neck space infections in this age group. ${ }^{4,8}$ In a review by Ruff et al, only 31 pediatric cases of infectious pseudoaneurysms of extracranial carotid artery had been reported since $1968 .^{9}$ Common organisms associated with this complication are Staphylococcus aureus and Streptococcus pyogenes. ${ }^{3}$ The mechanism of pseudoaneurysm formation is secondary to infectious arteritis of vessel wall with involvement of vasa vasorum, which results in ischemic vessel wall damage, subsequent erosion, and rupture. ${ }^{5}$

Children with extracranial ICA pseudoaneurysms usually present with painful pulsatile neck mass. Though thromboembolic events like stroke, transient ischemic attacks, or amaurosis fugax have been reported in adults, they are less commonly seen in children. ${ }^{10}$ Other complications which may be seen with mycotic ICA pseudoaneurysms include hemorrhage into aerodigestive tract secondary to rupture, airway compromise, and stridor due to mass effect, cranial nerve palsies, and Horner syndrome. Risk of rupture is more 


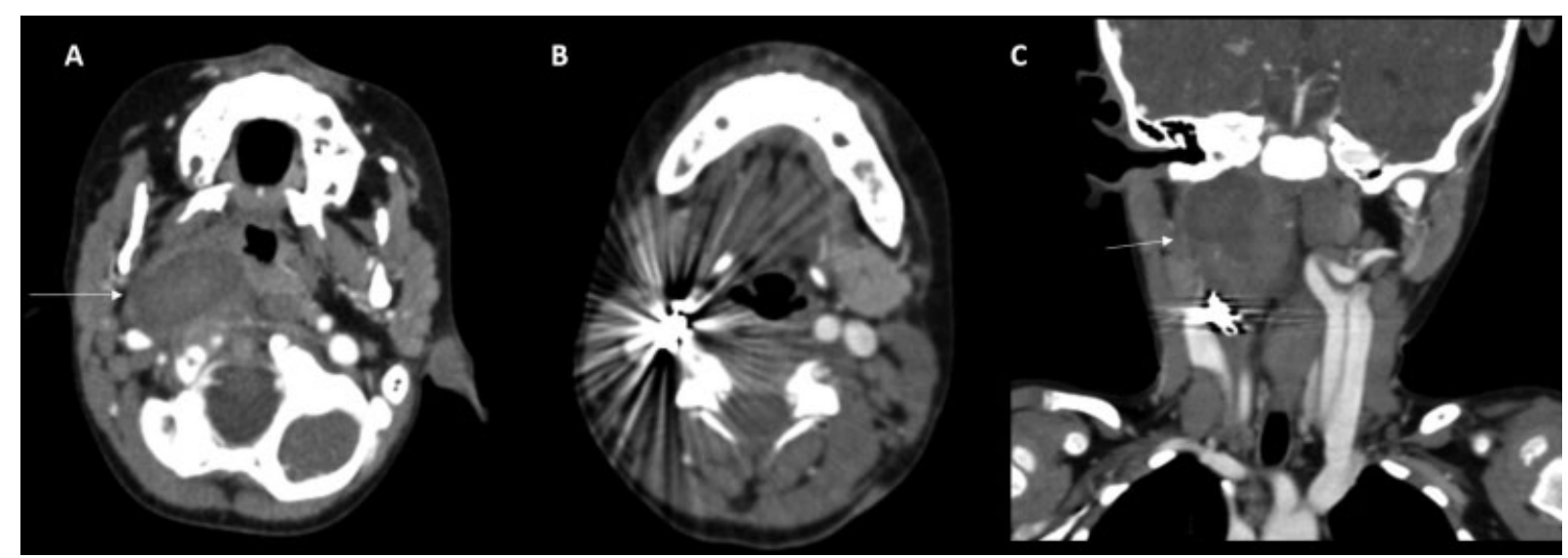

Fig. 3 Follow-up computed tomography (CT) angiography at 1 month showing complete thrombosis of pseudoaneurysm sac (white arrows in A and $\mathbf{C}$ ) with the coil mass (seen in B).

common in pseudoaneurysms in children, as compared with adults and in pseudoaneurysms occurring secondary to trauma and infections with high mortality rates in children (as high as $54 \%$ ). 3,11 Ultrasound is the initial imaging investigation of choice. Diagnosis is usually confirmed by CT angiography, though magnetic resonance angiography can be performed in older and cooperative children. ${ }^{12}$ Timely diagnosis and management is necessary to prevent morbidity and mortality from complications. ${ }^{5,13}$

There are no consensus guidelines till date and the choice of treatment depends on factors like site and etiology of pseudoaneurysm, and status of intracranial collateral circulation. ${ }^{4,14}$ Historically, surgery (under antibiotic cover) has been the mainstay of treatment. It offers a definitive cure but has a high complication rate including perioperative hemorrhage, Horner Syndrome, and cranial nerve injury (rates as high as 40\%) as well as mortality rates ranging from 9 to $20 \%{ }^{5,10}$ Surgery may also be technically difficult, especially in pseudoaneurysms near skull base and in small necks. ${ }^{1,3}$

Endovascular strategies have a lower mortality rate (4.1\%), lower risk of stroke (1.8\%) and cranial nerve injury (0.5\%), and shorter length of hospital stay. ${ }^{6,10}$ Endovascular strategies include PAO, coiling of pseudoaneurysm, using bare metal stents with/without coils, covered stents/stent grafts, and flow diverters to exclude the pseudoaneurysm from circulation. ${ }^{3}$ PAO using coils or detachable balloons is the preferred technique in children especially in emergencies, as they have good intracranial collateral circulation which is an essential prerequisite for PAO. ${ }^{2,4}$ Patency of stents and flow diverters with long-term antiplatelet medication is not well evaluated in children, hence making them less preferable in children. ${ }^{2,4,8}$ Bare metal stents, stent grafts, and flow diverters are not used in mycotic pseudoaneurysms, as they are prone to get infected and are not sufficient to prevent spread of septic emboli. In addition to lesion-specific management, the child should be kept on antimicrobial cover for 4 to 6 weeks. ${ }^{2,4,7}$ The probable complications of endovascular therapies include thromboembolism, arterial dissection, late stenosis, occlusion of stent graft, and recurrence. Immediate relief from compression-related symptoms may not be achieved with endovas- cular treatment. ${ }^{3,5}$ Long-term follow-up is essential to check for thrombosis and resolution of pseudoaneurysm, any recurrence, and patency of the stents. ${ }^{2}$

\section{Conclusion}

Mycotic pseudoaneurysms of ICA in children are rare, but potentially life-threatening complications of deep neck space infections requiring prompt management. PAO with trapping of neck of the aneurysm using endovascular coils or balloons is a safe and effective method of treatment. Longterm antimicrobial therapy and follow-up is vital to achieve optimal outcomes.

\section{Funding}

None.

Conflict of Interest

None declared.

\section{Acknowledgments}

None.

\section{References}

1 Garg K, Rockman CB, Lee V, et al. Presentation and management of carotid artery aneurysms and pseudoaneurysms. J Vasc Surg 2012;55(06):1618-1622

2 Balasundaram P, Sebastian LJD, Jain N, Prabhakar A, Garg A, Gaikwad S. Management of arterial pseudoaneurysms of the neck in a pediatric population: an endovascular case series and review of literature. World Neurosurg 2019;125:e273-e281

3 Chavan R, Ichaporia N, Vhora S, et al. Endovascular management of internal carotid artery pseudoaneurysms: retrospective observational study. Interdiscip Neurosurg 2021;24:101042

4 Kumar A, Prabhakar A, Gupta V, et al. Endovascular management of internal carotid artery pseudoaneurysms: a single-centre experience of 20 patients. Neurol India 2018;66(04):1067-1074

5 Brinjikji W, Diehn FE, Lindsay CW, Morris JM. Endovascular treatment of an infected pseudoaneurysm secondary to retropharyngeal abscess in a child. Interv Neuroradiol 2015;21(04):538-542

$6 \mathrm{Ni} \mathrm{L}$, Weng $\mathrm{H}, \mathrm{Pu} \mathrm{Z}$, et al. Open surgery versus endovascular approach in treatment of extracranial carotid artery aneurysms. J Vasc Surg 2018;67(05):1429-1437 
7 Thota R, Sebastian LJD, Gupta MM, et al. ICA pseudoaneurysms and fistulas presenting as severe epistaxis: endovascular management. Interdiscip Neurosurg 2020;22:100818

8 Nadarajah J, Sebastian LJD, Jain N, Gaikwad SB, Jauhari P, Saini A. Endovascular management of a rare case of pediatric vertebral artery mycotic aneurysm: a case report. Pediatr Neurosurg 2018; 53(05):346-350

9 Ruff MW, Nasr DM, Klaas JP, Renaud DL. Internal carotid artery pseudoaneurysm and ischemic stroke secondary to retropharyngeal and parapharyngeal abscess. J Child Neurol 2017;32(02): 230-236

10 Li Z, Chang G, Yao C, et al. Endovascular stenting of extracranial carotid artery aneurysm: a systematic review. Eur J Vasc Endovasc Surg 2011;42(04):419-426
11 Pourhassan S, Grotemeyer D, Fokou M, et al. Extracranial carotid arteries aneurysms in children: single-center experiences in 4 patients and review of the literature. J Pediatr Surg 2007;42(11): 1961-1968

12 Davidson C, Holihan C, de Oliveira Sillero R, Lee K, Mitchell RB, Shah G. Infectious pseudoaneurysm of the internal carotid artery in a child secondary to parapharyngeal abscess. Ear Nose Throat J 2021;:œ: 145561320984582

13 Salinger S, Pearlman SJ. Hemorrhage from pharyngeal and perionsillar abscesses. Arch Otolaryngol 1933;18(04):464-509

14 Sundarrajan C, Isa SA, Caruso JP, et al. Treatment of large infectious extracranial carotid artery pseudoaneurysms in children: a systematic review of the literature. Childs Nerv Syst 2021;37(05): 1461-1470 